\title{
Análisis de la producción científica de universidades en Comunicación Social (2014 - 2018)
}

\section{Analysis of the Scientific Production of Universities in Social Communication (2014 - 2018)}

Felipe Anderson Rios Incio

Universidad César Vallejo, Trujillo, Perú

ORCID: https://orcid.org/0000-0001-7049-8869

William Reynaldo Prado Morales

Universidad César Vallejo, Trujillo, Perú

ORCID: https://orcid.org/0000-0002-0136-4901

Alejandro Cruzata-Martínez*

Universidad San Ignacio de Loyola, Lima, Perú ORCID: https://orcid.org/0000-0003-0104-0496

\section{Sonia Cecilia Alvarado del Águila}

Universidad César Vallejo, Trujillo, Perú ORCID: https://orcid.org/0000-0003-0945-9259

Recibido 03-01-20 Revisado 13-03-20 Aprobado 20-04-20 En línea 18-06-20

*Correspondencia

Email: alejandrocruzatamartinez@yahoo.es
Citar como:

Rios, F., Prado, W., Cruzata-Martinez., A., \& Alvarado, C (2020). Análisis de la producción científica de universidades en Comunicación Social (2014 - 2018). Propósitos y Representaciones, 8(2), e558. doi: http://dx.doi.org/10.20511/pyr2020.v8n2.558 


\section{Resumen}

El presente artículo presenta un análisis de las investigaciones en Comunicación social durante los años 2014 - 2018 de estudiantes de pregrado en la ciudad de Trujillo; con el fin de determinar la orientación metodológica y temática se analizaron un total de 192 Tesis mediante una guía de análisis. Metodológicamente la investigación corresponde al enfoque positivista de diseño no experimental descriptivo comparativo. Los principales hallazgos en las universidades son la no evolución de las tesis de pre grado, siguiendo desde hace muchos años con un enfoque cuantitativo, de diseño no experimental con alcance descriptivo simple, siendo las áreas más trabajadas comunicación audiovisual, comunicología, diseño, publicidad y marketing.

Palabras clave: Comunicación; Trabajo de investigación; Enfoque científico; Análisis cuantitativo.

\section{Summary}

This document analyzes the research in Social Communication during the years 2014 - 2018 of undergraduate students in the city of Trujillo; In order to determine the methodological and thematic orientation, a total of 192 theses were analyzed using an analysis guide. Methodologically, the research corresponds to the positivist approach of comparative descriptive non-experimental design. The main findings in the universities are the non-evolution of undergraduate theses, following for many years with a quantitative approach, of non-experimental design with a simple descriptive scope, the most worked areas being audiovisual communication, communication, design, advertising and marketing.

Keywords: Communication, Research Work, Scientific Approach, Quantitative Analysis.

\section{Introducción}

La investigación científica en las universidades juega un papel importante en el desarrollo de la sociedad del conocimiento contemporáneo. No solo en la preparación formativa profesional de los estudiantes, sino además por su contribución desde las habilidades formativas como investigadores en los contextos sociales en lo que se desempeñarán, por lo cual se deberían desarrollar acciones orientadas a mejorar las competencias investigativas de los docenes y estudiantes (Larran-Jorge \& Andrades-Pena, 2015).

En el artículo $48^{\circ}$ del capítulo VI de la Ley Universitaria 30220, se refiere a la investigación como una función esencial y obligatoria de la universidad peruana; motivo por el cual se fomenta a realizar la investigación que responda a las necesidades de la realidad nacional, a través de la producción de conocimiento y desarrollo de nuevas tecnologías, agregando según Calderón, Gutiérrez y Castaño (2017) "la necesidad de articulación con el entorno y capacidad de realizar innovación" (p. 44-45)

Teniendo en cuenta, el argumento anterior, los docentes y los estudiantes, que se gradúen deben participar en la actividad investigativa de su universidad o en redes de investigación nacional o internacional, creadas por los centros superiores de estudios públicos o privados. Es por ello que Roque, García y Maldonado (2020), refieren "que la política científica institucional en la educación superior juega un importante papel dentro del proceso formativo en el nivel superior" (p.178).

En el Perú el estudiante de pregrado obtiene su título profesional presentando casi siempre una tesis y para Corrales, Pérez y Díaz (2019) una tesis universitaria "es una investigación que culmina los estudios de pregrado o posgrado, se caracteriza por ser inédita y original, permite consolidar la formación académica" (p.201). Esta investigación científica y su desarrollo 
didáctico en las universidades al ser trabajado desde la perspectiva docente con la utilización del método científico general de la ciencia, permite el rendimiento y eficiencia de la educación con un carácter holístico e integrador.

El rendimiento académico actualmente se ve reflejado en gran medida en la forma de cómo un individuo a partir de lo aprendido como resultado del proceso de formación posee capacidad de respuesta (Vergara, 2011). Esto debe ser consecuencia de un adecuado proceso de enseñanza- aprendizaje en los estudiantes, entendiendo que "para que una persona posea capacidad de producir conocimientos, y de aprendizaje permanente, se requiere tener capacidades para la investigación" (Miyahira, 2009, p.119), capacidades que deben de generarse de forma gradual y holística en el proceso de enseñanza-aprendizaje de las universidades, reflejado en las investigaciones de grado de las mismas.

La eficiencia de las universidades debe medirse entre otras formas por el aporte de las investigaciones a la comunidad (García de Fanelli, 2000) (Ibáñez, Morresi \& Delbianco, 2017). Debe entenderse que una universidad que no genere nuevos conocimientos a partir de sus procesos de investigación, no es una universidad (Pétriz \& Rubiralta, 2017). Es así que al formar el proceso de investigación algo importante, fundamental y sobre todo misional en las universidades, las direcciones de investigación de las mimas deben planificar estratégicamente sus actividades para lograr el mayor rendimiento y eficiencia en los procesos de enseñanza aprendizaje, para lo cual se requiere directivos con competencias metodológicas e investigativas (Sánchez, Sánchez \& Viloria, 2016-2017)

En cuanto a la investigación científica en nuestro país Bermúdez (2013), asegura que en la mayoría de las universidades del Perú no están en los niveles de productividad ni calidad que la modernidad exige, debido a una falla en la orientación de formación de una gran cantidad de profesionales y generación de rentabilidad económica. La cruda realidad es que tanto en las universidades, tanto públicas y privadas del país, no investigan, y si lo hacen no hay una buena orientación y, por consiguiente, no producen ciencia y tecnología, existiendo así problemas en la investigación (Huaraca, Apaza y Mejía, 2017). Ante esta situación es necesario iniciar en el Perú el verdadero proceso de alfabetización científica y tecnológica que parte desde los centros de educación superior universitaria.

Mejorar el rendimiento y la eficiencia en la educación reflejada en la calidad educativa en las universidades debe integrar precisamente objetivos relacionados entre otros con el proceso de investigación como método fundamental, de manera concreta, tanto en la institución como en el ámbito social donde se desenvuelven durante su quehacer universitario (Ruiz y López, 2019) (Ramírez, García y Cruel, 2017), para ellos debería verse a la investigación como un proceso holístico orientado a la generación de nuevos conocimientos utilizando los diversos paradigmas que esta posee.

En las universidades peruanas de forma general y en particular, las de la región La Libertad, existe desde la didáctica de la metodología de la investigación científica, la tendencia, sólo al desarrollo de trabajos de investigación desde el enfoque cuantitativo. No obstante, desde el análisis de la literatura consultada, se identifican además de los enfoques cuantitativos, los cualitativos y holísticos que conllevan a mejores resultados desde la complementación entre los diferentes métodos en el desarrollo de las investigaciones científicas, tal como lo afirma Pereira (2011) cuando indica "que los diseños mixtos han ido cobrando fuerza día a día y cada vez son más aplicados en investigaciones en diversos campos, en especial, dentro de las Ciencias Sociales" (p.16). Aplicar la metodología anteriormente expuesta implica un impacto positivo en el proceso formativo e investigativo de los profesionales desde las universidades, obligando según Pineda (2006) a la "experimentación metodológica como vía para poder combinar creativamente enfoques cuantitativos, cualitativos, teóricos y empíricos de manera que sea posible un conocimiento más integral de lo que es la comunicación hoy y a futuro" (p.156). 
Y es que "la formación investigativa es un asunto complejo que obligatoriamente exige abordarse desde didácticas diferentes a las del método tradicional, imperante en muchas instituciones de educación superior" (Córdoba, 2016, p.22). Gutiérrez, Benítez, y González (2018) "La universidad moderna está evolucionando desde sus orígenes académicos convencionales hacia un ámbito cada vez más profesional, razones que obligan al establecimiento de enfoques no tradicionales en su desarrollo (p.49-50), es por ello que "se hace indispensable redoblar los esfuerzos por elaborar sistemas de categorías descriptivas suficientemente consistentes para sistematizar las tendencias temático-referenciales y teórico-metodológicas que orientan los productos de la investigación" (Navarro, 2011, p.227).

Sin embargo, es preciso indicar que las metodologías a utilizar en las investigaciones en Ciencias de la Comunicación se encuentran estrechamente afines con lo que el investigador pueda y quiera observar, con la mirada que este le dé a su trabajo, de modo que un problema de investigación determinado requiere una estrategia metodológica determinada.

En las llamadas Ciencias de la Comunicación, han existido desde siempre estrategias metodológicas que han dominado las investigaciones, las cuales han sido validadas por los centros de investigación y otros sectores de la academia y la sociedad (Fuentes, 1999).

En la ciudad de Trujillo se han desarrollado en mayor proporción las metodologías cuantitativas, en un repaso general de las tesis presentadas en el 2018 por la escuela de Ciencias de la Comunicación de la Universidad César Vallejo, encontramos que el 85\% corresponde a esta metodología (encuestas por muestreo, estudios de audiencia y de opinión, análisis de contenido de medios, planes de comunicación, etc.).

Como consecuencia de lo anteriormente expuesto, es interesante entonces formularse como pregunta de investigación: ¿Cuál es la orientación metodológica y temática de las investigaciones de tesis en Ciencias de la Comunicación por parte de los estudiantes de la Universidad Nacional de Trujillo, Universidad César Vallejo y Universidad Privada Antenor Orrego en el período que abarca desde el año 2014 al $2018 ?$

De tal manera que conozcamos en qué estado se encuentran estas investigaciones, es principal objetivo de la investigación determinar la orientación metodológica y temática de las investigaciones de tesis en Ciencias de la Comunicación por parte de los estudiantes de pregrado. Después de contextualizar el problema de investigación, es necesario realizar una fundamentación teórica. Es por ello que a continuación se analizarán las bases teóricas que sustentan el trabajo de investigación desarrollado.

Una de las variables de estudio tenida en cuenta, es la metodología de la investigación, para la cual Hernández, Fernández y Baptista (2014) concibe a la investigación científica como "un conjunto de procesos sistemáticos y empíricos que se aplican al estudio de un fenómeno; es dinámica, cambiante y evolutiva" (p.25).

Es importante indicar que en lo que va de la historia de la humanidad y la ciencia, en su trayecto de esta última han nacido distintas corrientes de pensamiento científico, como el empirismo, positivismo, algunas otras corrientes como la etnografía, el estructuralismo y demás, que han originado finalmente diversas orientaciones en la búsqueda del conocimiento. Así es como se origina en la investigación científica dos corrientes principales; el enfoque cuantitativo y el enfoque cualitativo.

Grinnell (1997) manifiesta que los enfoques cualitativos y cuantitativos utilizan en su desarrollo procesos cuidadosos, ordenados y experimentales en su pretensión de crear conocimiento, razón por la cual, la conceptualización previa de investigación se emplea a los dos enfoques igualitariamente, y desarrollan cinco etapas afines y congruentes entre sí. 
Lo ideal sería que, en las universidades, se practique la investigación aplicada para contribuir con el desarrollo social; sin embargo, González (2013), asegura que -a nivel mundiales casi imposible mantener el equilibrio entre la investigación básica y la aplicada, debido al alto costo de inversión que demanda la investigación aplicada, y más aún si se trata de vincularla al conocimiento de ámbito mundial con los problemas locales, situación que también estaría ocurriendo en nuestro medio.

Otra de las variables de estudio es la temática en la investigación. Al consultar los temas de las tesis desarrolladas en los últimos años por los estudiantes de Ciencias de la Comunicación de la Universidad César Vallejo (Trujillo), se llegó a determinar que un $40 \%$ de las mencionadas investigaciones proponen planes de comunicación para determinadas empresas, lo cual implica trabajar en beneficio de determinada empresa, más no de la sociedad en su conjunto de manera holística.

La UNESCO (1998), en la declaración mundial de educación superior sostiene la urgente necesidad que la educación universitaria y no universitaria de los diversos países del mundo, responda a los retos impuestos por la globalización, y para ello, hay que utilizar redes, transmitir uso de tecnología moderna, crear nuevos escenarios pedagógicos, aplicar la tecnología a las necesidades regionales y nacionales, continuar con la gestión del conocimiento, favorecer la colaboración de modo internacional para actualizar las actividades académicas e innovar, factor crucial en el desarrollo y pertenencia de la educación superior y de la economía de cualquier nación.

Entonces queda claro que, a nivel mundial, hay una preocupación por alinear los conocimientos impartidos en las universidades, con el desarrollo de cada región. Precisamente en el Congreso Iberoamericano de Ciencia, Tecnología, Innovación y Educación, desarrollado en el 2014 en Argentina, se concluyó que el conocimiento científico es la expresión del espíritu humano; y, por lo tanto, es el insumo necesario para el desarrollo económico y social.

Por lo expuesto anteriormente, la UNESCO indica que el conocimiento es una de las principales riquezas de las sociedades contemporáneas, de ahí que la prosperidad de las naciones está asociada al valor que agrega el conocimiento a la producción de bienes y servicios.

Además en el referido Congreso también se precisó que la "sociedad del conocimiento" y "economía del conocimiento", el éxito de una sociedad depende -en gran medida- de la excelente formación en las universidades de los profesionales, quienes deben tener el conocimiento y la experiencia para desarrollar cambios tecnológicos en la sociedad, los mismos que deben ser aplicados a la realidad, cuidando y mejorando los recursos naturales, la salud, la desnutrición, la falta de oportunidades en empleo, educación y otros requerimientos que la sociedad necesita, los cuales se verán reflejados en sus investigaciones de grado.

En el campo de la Comunicación, Arévalo y Farné (2016) han realizado un análisis en España, donde han determinado un creciente interés por profundizar en el estudio de la comunicación y el cambio social. Dicho crecimiento se expresa en el desarrollo de proyectos de investigación, la publicación de artículos, libros y capítulos, la organización de congresos y seminarios, el funcionamiento de redes de trabajo o la aplicación de este conocimiento en campañas de sensibilización, entre otros aspectos.

Elbirt (2013) indica que "las Ciencias de la Comunicación integran diferentes disciplinas y perspectivas teóricas, así como también, metodologías y herramientas de intervención social" (P.66). Entre las especialidades que desarrollan las universidades y facultades de comunicación en Trujillo, están la de audiovisuales, el periodismo, comunicación corporativa, la Comunicación para el desarrollo, una especialidad muy importante para el desarrollo armónico de la comunidad internacional, motivo por el cual la Organización de las Naciones Unidas (2011), ha entendido el 
papel que la comunicación puede desempeñar en el empoderamiento de las personas para influir sobre las decisiones que afectan a sus vidas.

Por lo tanto, la comunicación para el desarrollo (CPD, Communication for Development o C4D por sus siglas en inglés) es percibida por la ONU, como uno de los modos más importantes para ampliar el acceso a estas nuevas oportunidades.

Tal es la importancia de la CPD, que la ONU la considera como un proceso social, que fomenta el diálogo entre las comunidades y los responsables de la adopción de decisiones locales, nacionales y regionales.

Para Gumucio (2011), el aporte de América Latina en los estudios de comunicación es ser pionera en la comunicación para el desarrollo y como pionera en la generación tanto de prácticas concretas como de pensamiento crítico.

Se ha desarrollado experiencias interesantes a nivel nacional, regional y local, y a partir de las conclusiones de diversas investigaciones, se ha corroborado que una comunicación que gira sobre la participación ciudadana, se convierte en una comunicación realmente participativa, basada en el diálogo de los participantes, avala el desarrollo social que toda comunidad requiere. Entonces es importante que, en las Facultades de Comunicación, se fomente desarrollo e implementación de políticas y programas que mejoren la calidad de vida de todas las personas.

Otras de las especialidades en ciencias de la comunicación que se desarrollan en las facultades de Trujillo, es el diseño y la publicidad: Ciencias de la Comunicación tiene una estrecha relación con la Publicidad, en este sentido, Tena (2017) sostiene que el objetivo principal de la publicidad es establecer un plan orientado a resolver, a corto plazo, los problemas de comunicación de un cliente; de allí la importancia de dominar las técnicas del diseño publicitario. La línea de investigación según Agudelo (2004) es "un campo temático, es una perspectiva sistemática y exhaustiva en la generación de nuevo conocimiento" (p.11) y debe de poseer racionalidad que permita la integración de la comunidad científica en el desarrollo específico del conocimiento en torno a un eje temático común.

En las Universidades de Trujillo se constataron las líneas designadas bajo resoluciones rectorales, como: Procesos comunicacionales en la sociedad contemporánea, comunicación para el desarrollo humano, Comunicación, sociedad y cultura, Comunicación y organizaciones, Nuevas tecnologías y discursos de la comunicación, educomunicación, entre otras, esto demuestra que las facultades de Comunicación y las universidades que las albergan han hecho un esfuerzo por ordenar la actividad investigativa.

Igartua y Humanes (2004) nos indican que "cuando hablamos de teorías de la comunicación nos estamos refiriendo a un cuerpo de conocimientos que se asienta en un análisis científico social del objeto de la comunicación" (p.2), y estas teorías deberían forjar las bases y el rumbo teórico de las investigaciones.

El presente trabajo de investigación se justifica desde el punto de vista teórico, debido a que permitirá consultar y recordar los principales puntos de la Ley Universitaria 30220, y su relación directa con la teoría de cómo debe ser un centro superior de estudios, y sobre todo, lo que espera la comunidad de los profesionales que se forman.

Sin duda el presente trabajo de investigación contribuye en la relación de varios conceptos y teorías con la Ley Universitaria peruana, que incita a la mejora continua de la educación, con énfasis en las instituciones de educación superior universitaria como entes fundamentales en el desarrollo del país, de la academia e investigación y de la cultura. 
Desde el punto de vista metodológico, en el estudio propuesto, se utilizó la comparación, que permitió determinar qué Facultad de Ciencias de la Comunicación de la ciudad de Trujillo cumple con los principios básicos de investigación impuesto por la Ley Universitaria u orienta mejor a sus estudiantes.

Desde el punto de vista práctico, el presente estudio tiene un aporte valioso porque a través de la observación y comparación se determinó el rumbo que los estudiantes han tomado en cada una de las Facultades estudiadas.

La relevancia de la investigación propuesta radica fundamentalmente en su compromiso y responsabilidad social en la que se permite contribuir en el ámbito educativo de las Facultades de Ciencias de Comunicación de Trujillo, ya que el estudio comparativo permitió conocer la realidad de las investigaciones, y de ser necesario, reorientar los temas de investigación de los estudiantes para que se conviertan en reales entidades académicas orientadas a la investigación científica, que brinda una formación orientada en la corriente humanista, científica y con énfasis en lo tecnológico con una clara concientización de la realidad multicultural de nuestro país.

\section{Método}

La presente investigación tiene un enfoque cuantitativo con un diseño transversal descriptivo comparativo. Se logró analizar un total de 192 de las 365 tesis correspondientes a tres universidades de la ciudad de Trujillo durante los períodos del 2014 al 2018, se utilizó el muestreo estratificado que busca respetar para la muestra esa distribución balanceada de la población, utilizando criterios de inclusión y exclusión.

Para el recojo de información primaria, se diseñó como técnica de investigación principal el análisis de contenido y como instrumento la lista de cotejo, que según Tobón (2013) la define como una tabla de doble entrada con posibilidades de evaluar los indicadores, es útil cuando se tienen grandes muestras, pero a la vez muchas veces presenta desventajas cuando no tiene puntos intermedios para una determinada evaluación.

Asimismo, la entrevista, que según Denzin y Lincoln (2005) la definen como una conversación que consiste en realizar preguntas al entrevistado y recibir respuesta del mismo.

Para el análisis y tratamiento de la información se procesaron los resultados encontrados en software SPSS y Excel 2016, los resultados se presentaron en cuadros utilizando estadística descriptiva. Se realizó un comparativo por Universidad, el resultado de las entrevistas por parte de los docentes sirvió para profundizar y reforzar los hallazgos, considerando antecedentes y marco teórico en la discusión de los resultados.

\section{Resultados}

Los resultados presentados a continuación constituyen el análisis documental realizado a 192 tesis de estudiantes de pre grado en Ciencias de la Comunicación de tres universidades en la ciudad de Trujillo, tal como se observa en la Tabla 1. 
Tabla 1.

Estratificación de tesis por Universidad (2014 - 2018)

\begin{tabular}{ccccccc}
\hline Universidad & 2014 & 2015 & 2016 & 2017 & 2018 & Total \\
\hline Universidad César Vallejo & 17 & 24 & 26 & 32 & 23 & 122 \\
Universidad Privada Antenor & 9 & 9 & 16 & 14 & 9 & 57 \\
$\begin{array}{c}\text { Orrego } \\
\text { Universidad Nacional de }\end{array}$ & 3 & 2 & 3 & 3 & 2 & 13 \\
$\begin{array}{c}\text { Trujillo } \\
\text { TOTAL }\end{array}$ & 29 & 35 & 44 & 49 & 34 & 192 \\
\hline
\end{tabular}

Fuente: Estadísticas presentadas de manera oficial por las universidades (enero 2019)

En cuanto a la metodología utilizada por los estudiantes de Pregrado de las universidades investigadas se corroboró que las tesis en su mayoría son de enfoque cuantitativo, siendo la Universidad César Vallejo con el $80 \%$ de ellas, la universidad que mayor tesis de enfoque cuantitativo han desarrollado sus estudiantes. El diseño utilizado por las 3 universidades de Trujillo ha sido el no experimental con alcance descriptivo simple, la Universidad Privada Antenor Orrego desarrolló el 95\% de tesis con el diseño ya mencionado.

Además, en el estudio, se ha determinado que la encuesta es la técnica de investigación más utilizada por los estudiantes de las tres universidades de Trujillo. En la Universidad Privada Antenor Orrego, el $50 \%$ de estudiantes utilizan esta técnica, seguido de la observación con un $28 \%$ en la Universidad Nacional de Trujillo, y la entrevista con un $20 \%$ en las tres universidades, cada técnica con sus respectivos instrumentos de investigación, siendo el cuestionario el instrumento más utilizado por la Universidad Privada Antenor Orrego con un 45\%, seguido de la guía de observación de la misma universidad con $25 \%$ y la guía de entrevista con $20 \%$ en las universidades César Vallejo y Nacional de Trujillo. Finalmente, para el procesamiento y análisis de datos los estudiantes de la Universidad César Vallejo utilizan excel en un 47\% (ver tabla 2). 
Tabla 2.

Comparativo metodológica de las tesis (2014 - 2018)

\begin{tabular}{|c|c|c|c|c|c|c|}
\hline \multirow{3}{*}{ Indicadores } & \multicolumn{2}{|c|}{ UCV } & \multicolumn{2}{|c|}{ UNT } & \multicolumn{2}{|c|}{ UPAO } \\
\hline & & Porcen & & Porcen & & Porcen \\
\hline & $\mathrm{F}$ & taje & $\mathrm{f}$ & taje & $\mathrm{f}$ & taje \\
\hline \multicolumn{7}{|l|}{ Enfoque } \\
\hline Cuantitativo & 97 & $80 \%$ & 8 & $62 \%$ & 43 & $75 \%$ \\
\hline Cualitativo & 17 & $14 \%$ & 2 & $15 \%$ & 4 & $7 \%$ \\
\hline Mixto & 4 & $3 \%$ & 2 & $15 \%$ & 9 & $16 \%$ \\
\hline No indica & 4 & $3 \%$ & 1 & $8 \%$ & 1 & $2 \%$ \\
\hline Total & 122 & $100 \%$ & 13 & $100 \%$ & 57 & $100 \%$ \\
\hline \multicolumn{7}{|l|}{ Diseño } \\
\hline Experimental & 23 & $19 \%$ & 2 & $15 \%$ & 1 & $2 \%$ \\
\hline No experimental & 92 & $75 \%$ & 11 & $85 \%$ & 54 & $95 \%$ \\
\hline No indica & 7 & $6 \%$ & 0 & $0 \%$ & 2 & $4 \%$ \\
\hline Total & 122 & $100 \%$ & 13 & $100 \%$ & 57 & $100 \%$ \\
\hline \multicolumn{7}{|l|}{ Alcance } \\
\hline Descriptivo & 53 & $43 \%$ & 6 & $43 \%$ & 38 & $67 \%$ \\
\hline Descriptivo propositivo & 14 & $11 \%$ & 1 & $7 \%$ & 1 & $2 \%$ \\
\hline Descriptivo comparativo & 6 & $5 \%$ & 0 & $0 \%$ & 4 & $7 \%$ \\
\hline Explicativo & 2 & $2 \%$ & 0 & $0 \%$ & 9 & $16 \%$ \\
\hline Correlacional & 17 & $14 \%$ & 2 & $14 \%$ & 4 & $7 \%$ \\
\hline Quasi experimental & 0 & $0 \%$ & 1 & $7 \%$ & 0 & $0 \%$ \\
\hline Pre experimental & 20 & $16 \%$ & 1 & $7 \%$ & 1 & $2 \%$ \\
\hline Etnográfico & 0 & $0 \%$ & 1 & $7 \%$ & 0 & $0 \%$ \\
\hline Fenomenológico & 0 & $0 \%$ & 2 & $14 \%$ & 0 & $0 \%$ \\
\hline Investigación acción & 6 & $5 \%$ & 0 & $0 \%$ & 0 & $0 \%$ \\
\hline Otros & 2 & $2 \%$ & 0 & $0 \%$ & 0 & $0 \%$ \\
\hline No indica & 2 & $2 \%$ & 0 & $0 \%$ & 0 & $0 \%$ \\
\hline Total & 122 & $100 \%$ & 14 & $100 \%$ & 57 & $100 \%$ \\
\hline \multicolumn{7}{|l|}{ Técnicas } \\
\hline Encuesta & 81 & $42 \%$ & 9 & $36 \%$ & 46 & $50 \%$ \\
\hline Observación & 42 & $22 \%$ & 7 & $28 \%$ & 19 & $21 \%$ \\
\hline Entrevista & 39 & $20 \%$ & 5 & $20 \%$ & 18 & $20 \%$ \\
\hline Análisis de contenido & 14 & $7 \%$ & 2 & $8 \%$ & 0 & $0 \%$ \\
\hline Análisis documental & 2 & $1 \%$ & 0 & $0 \%$ & 0 & $0 \%$ \\
\hline Focus & 11 & $6 \%$ & 0 & $0 \%$ & 7 & $8 \%$ \\
\hline Otras & 4 & $2 \%$ & 0 & $0 \%$ & 0 & $0 \%$ \\
\hline No indica & 1 & $1 \%$ & 2 & $8 \%$ & 2 & $2 \%$ \\
\hline TOTAL & 194 & $100 \%$ & 25 & $100 \%$ & 92 & $100 \%$ \\
\hline \multicolumn{7}{|l|}{ Instrumento } \\
\hline Cuestionario & 83 & $43 \%$ & 6 & $24 \%$ & 46 & $45 \%$ \\
\hline Guía de observación & 42 & $22 \%$ & 5 & $20 \%$ & 26 & $25 \%$ \\
\hline Lista de cotejo & 1 & $1 \%$ & 1 & $4 \%$ & 3 & $3 \%$ \\
\hline Guía de entrevista & 39 & $20 \%$ & 5 & $20 \%$ & 18 & $18 \%$ \\
\hline Guía de análisis de contenido & 16 & $8 \%$ & 1 & $4 \%$ & 0 & $0 \%$ \\
\hline Guía de análisis documental & 1 & $1 \%$ & 0 & $0 \%$ & 0 & $0 \%$ \\
\hline Formato de autoevaluación & 1 & $1 \%$ & 0 & $0 \%$ & 0 & $0 \%$ \\
\hline Guía de discusión grupal & 11 & $6 \%$ & 0 & $0 \%$ & 7 & $7 \%$ \\
\hline Otros & 0 & $0 \%$ & 5 & $20 \%$ & 0 & $0 \%$ \\
\hline No indica & 0 & $0 \%$ & 2 & $8 \%$ & 2 & $2 \%$ \\
\hline TOTAL & 194 & $100 \%$ & 25 & $100 \%$ & 102 & $100 \%$ \\
\hline \multicolumn{7}{|c|}{ Procesamiento y análisis de datos } \\
\hline Excel & 57 & $47 \%$ & 4 & $27 \%$ & 32 & $46 \%$ \\
\hline SPSS & 18 & $15 \%$ & 4 & $27 \%$ & 23 & $33 \%$ \\
\hline No indica & 47 & $39 \%$ & 7 & $47 \%$ & 14 & $20 \%$ \\
\hline TOTAL & 122 & $100 \%$ & 15 & $100 \%$ & 69 & $100 \%$ \\
\hline
\end{tabular}


En cuanto a la orientación temática de las investigaciones de pregrado se puede encontrar en la Tabla 3 que el 23\% de tesis Ciencias de la Comunicación de la Universidad César Vallejo corresponde a la especialidad de diseño, publicidad y marketing, seguido de la especialidad de Comunicación Audiovisual con un 20\%, asimismo se encuentra que el $31 \%$ de las tesis de la Universidad Nacional de Trujillo corresponden a las especialidades de Comunicación Audiovisual y Comunicología y por último el 35\% de las tesis de la Universidad Privada Antenor Orrego corresponden área de diseño, publicidad y marketing seguida con un $32 \%$ la especialidad de Comunicación Corporativa.

En cuanto a las líneas de investigación se puede observar en la Tabla 3 que el 95\% de las tesis de la Universidad César Vallejo corresponde a la línea de investigación de Procesos comunicacionales en la sociedad contemporánea, el 100\% de las tesis de la Universidad Nacional de Trujillo y Universidad Privada Antenor Orrego no indican a qué línea de investigación están orientados sus trabajos de grado.

Finalmente, el $49 \%$ de las investigaciones de la Universidad César Vallejo no indican en qué teorías de la comunicación se han basado para desarrollar su marco teórico, solo el $8 \%$ de los tesistas utilizó la teoría de usos y gratificaciones y el 5\% la teoría del desarrollo humano, el 57\% de las investigaciones de la Universidad Nacional de Trujillo no indican en qué teoría de la comunicación se han basado para desarrollar su marco teórico, solo el 3\% de los tesistas utilizó la teoría de estudios culturales y teoría de usos y gratificaciones. 
Tabla 3.

Comparativo temático de las tesis (2014 - 2018)

\begin{tabular}{|c|c|c|c|c|c|c|}
\hline \multirow[b]{2}{*}{ Indicadores } & \multicolumn{2}{|c|}{ UCV } & \multicolumn{2}{|c|}{ UNT } & \multicolumn{2}{|c|}{ UPAO } \\
\hline & $\mathrm{F}$ & $\begin{array}{c}\text { Porcen } \\
\text { taie }\end{array}$ & $\mathrm{f}$ & $\begin{array}{c}\text { Porcen } \\
\text { taie }\end{array}$ & $f$ & $\begin{array}{c}\text { Porcen } \\
\text { taie }\end{array}$ \\
\hline \multicolumn{7}{|l|}{ Área de especialidad } \\
\hline Periodismo & 16 & $13 \%$ & 1 & $8 \%$ & 5 & $9 \%$ \\
\hline $\begin{array}{l}\text { Diseño, Publicidad y } \\
\text { Marketing }\end{array}$ & 28 & $23 \%$ & 0 & $0 \%$ & 20 & $35 \%$ \\
\hline $\begin{array}{l}\text { Comunicación Audiovisual } \\
\text { (fotografía, radio, televisión y } \\
\text { cine) }\end{array}$ & 25 & $20 \%$ & 4 & $31 \%$ & 11 & $19 \%$ \\
\hline Comunicación Corporativa & 11 & $9 \%$ & 2 & $15 \%$ & 18 & $32 \%$ \\
\hline $\begin{array}{l}\text { Comunicación para el } \\
\text { desarrollo }\end{array}$ & 17 & $14 \%$ & 1 & $8 \%$ & 2 & $4 \%$ \\
\hline Medios digitales & 16 & $13 \%$ & 1 & $8 \%$ & 1 & $2 \%$ \\
\hline Comunicología & 4 & $3 \%$ & 4 & $31 \%$ & 0 & $0 \%$ \\
\hline No se adapta a la especialidad & 5 & $4 \%$ & 0 & $0 \%$ & 0 & $0 \%$ \\
\hline TOTAL & 122 & $100 \%$ & 13 & $100 \%$ & 57 & $100 \%$ \\
\hline \multicolumn{7}{|l|}{ Línea de investigación } \\
\hline $\begin{array}{l}\text { Procesos comunicacionales } \\
\text { en la sociedad contemporánea }\end{array}$ & 116 & $95 \%$ & 0 & $0 \%$ & 0 & $0 \%$ \\
\hline $\begin{array}{l}\text { Comunicación para el } \\
\text { desarrollo humano }\end{array}$ & 5 & 4 & 0 & 0 & 0 & 0 \\
\hline Otra sin resolución & 1 & $1 \%$ & 0 & $0 \%$ & 0 & $0 \%$ \\
\hline No indica & 0 & $0 \%$ & 13 & $100 \%$ & 47 & $100 \%$ \\
\hline TOTAL & 122 & $100 \%$ & 13 & $100 \%$ & 47 & $100 \%$ \\
\hline \multicolumn{7}{|l|}{ Teorías utilizadas } \\
\hline $\begin{array}{l}\text { Teoría de la aguja } \\
\text { hipodérmica }\end{array}$ & 4 & $3 \%$ & 0 & $0 \%$ & 0 & $0 \%$ \\
\hline Teoría de la agenda setting & 2 & $2 \%$ & 0 & $0 \%$ & 0 & $0 \%$ \\
\hline Modelo cultural de Deninson & 0 & $0 \%$ & 0 & $0 \%$ & 2 & $3 \%$ \\
\hline $\begin{array}{l}\text { Teoría de usos y } \\
\text { gratificaciones }\end{array}$ & 10 & $8 \%$ & 0 & $0 \%$ & 2 & $3 \%$ \\
\hline Teoría crítica & 3 & $2 \%$ & 0 & $0 \%$ & 0 & $0 \%$ \\
\hline Teoría de los Stakeholders & 2 & $2 \%$ & 0 & $0 \%$ & 0 & $0 \%$ \\
\hline Teoría de estudios culturales & 0 & $0 \%$ & 2 & $14 \%$ & 2 & $3 \%$ \\
\hline $\begin{array}{l}\text { Teoría de la sociedad de la } \\
\text { información }\end{array}$ & 4 & $3 \%$ & 2 & $14 \%$ & 0 & $0 \%$ \\
\hline Teoría de la mediación social & 1 & $1 \%$ & 0 & $0 \%$ & 0 & $0 \%$ \\
\hline Teoría del desarrollo humano & 7 & $5 \%$ & 0 & $0 \%$ & 0 & $0 \%$ \\
\hline Teoría de la imagen & 0 & $0 \%$ & 0 & $0 \%$ & 2 & $3 \%$ \\
\hline Teoría de Gatekeeper & 5 & $4 \%$ & 0 & $0 \%$ & 0 & $0 \%$ \\
\hline Teoría del espiral del silencio & 1 & $1 \%$ & 0 & $0 \%$ & 0 & $0 \%$ \\
\hline $\begin{array}{l}\text { Teoría de las relaciones } \\
\text { públicas }\end{array}$ & 5 & $4 \%$ & 0 & $0 \%$ & 0 & $0 \%$ \\
\hline Otras & 36 & $28 \%$ & 4 & $29 \%$ & 18 & $30 \%$ \\
\hline No indica & 49 & $38 \%$ & 6 & $43 \%$ & 34 & $57 \%$ \\
\hline TOTAL & 129 & $100 \%$ & 14 & $100 \%$ & 60 & $100 \%$ \\
\hline
\end{tabular}




\section{Discusión}

Para analizar la orientación metodológica y temática de las investigaciones de tesis en Ciencias de la Comunicación por parte de los estudiantes de la Universidad Nacional de Trujillo, Universidad César Vallejo y Universidad Privada Antenor Orrego en el período que abarca desde el año 2014 al 2018, los resultados se discutieron en función a los objetivos de investigación, basándose en los hallazgos que se contrastan con las fuentes bibliográficas y teóricas.

Las muestras de tesis corresponden a 122 tesis de la Universidad César Vallejo, 57 tesis de la Universidad Privada Antenor Orrego y 13 tesis de la Universidad Nacional de Trujillo (Tabla $1)$.

La investigación o el proceso de investigación, se sustenta en informaciones obtenidas directamente de la realidad, que le permiten al investigador verificar las condiciones reales de la obtención de los datos. Por lo que las investigaciones de las tesis en Comunicación pueden dividirse en enfoques cuantitativo (positivista), cualitativo (naturalista) y mixto (socio crítico).

Grinnell (1997) manifiesta que los enfoques cualitativos y cuantitativos utilizan en su desarrollo procesos cuidadosos, ordenados y experimentales en su pretensión de crear conocimiento, razón por la cual, la conceptualización previa de investigación se emplea a los dos enfoques igualitariamente, cosa que no ocurre en las investigaciones analizadas donde encontramos diseños de investigación que no corresponden los enfoques de investigación, tesis cualitativas con variables de investigación, investigaciones correlaciones con variables dependientes e independientes, tesis cualitativas donde se operacionaliza las variables, confusión entre las técnicas e instrumentos de investigación, entre otros.

En la Tabla 2 podemos notar que el $62 \%$ de tesis de Ciencias de la Comunicación de la Universidad Nacional de Trujillo son de enfoque cuantitativo, el $85 \%$ de tesis corresponden al diseño no experimental, el alcance de la mayoría de las tesis se encuentra el método al descriptivo simple con un $45 \%$. En la Tabla $\mathrm{N}^{\circ} 4$. El $80 \%$ de tesis Ciencias de la Comunicación de la Universidad César Vallejo son de enfoque cuantitativo, el $75 \%$ de tesis corresponden al diseño no experimental, el alcance de la mayoría de las tesis encontramos al descriptivo simple con un 43\%. En la Tabla $\mathrm{N}^{\circ} 6$. El 75\% de tesis Ciencias de la Comunicación de la Universidad Privada Antenor Orrego son de enfoque cuantitativo, el $95 \%$ de tesis corresponden al diseño no experimental, el alcance de la mayoría de las tesis se centra en el descriptivo simple con un $67 \%$. Esto concuerda con las investigaciones de Vera, Castañeda y Chirinos (2015) y Carrión (2018) quienes encontraron las tesis de los estudiantes de Ciencias de la Comunicación un enfoque cuantitativo, en su mayoría con diseño no experimental descriptivo, lo que indica que la tendencia desde el año 1996 no ha cambiado ni orientado a tesis de otro enfoque.

Las tesis de Ciencias de la Comunicación siguen siendo de Nivel básico, y esto se sustenta en lo indicado por González (2013), quien asegura que -a nivel mundial- es casi imposible mantener el equilibrio entre la investigación básica y la aplicada, debido al alto costo de inversión que demanda la investigación aplicada, y más aún si se trata de vincularla al conocimiento de ámbito mundial con los problemas locales, situación que también estaría ocurriendo en nuestro medio. Sin duda, las investigaciones realizadas por los estudiantes de Ciencias de Comunicación de las tres universidades trujillanas en estudios, se quedaron en el modelo tradicional. No han tomado en cuenta que el escenario de aprendizaje e investigación, se transforma vertiginosamente, lo cual implica la revisión de los diseños formativos implantados, por otros que respondan a las necesidades de la persona, ciudadano y profesional del siglo 21 (Ruiz-Corbella \& López-Gómez, 2019). Por otro lado, Bermúdez (2013) asegura que en la mayoría de las universidades del Perú no están en los niveles de productividad ni calidad que la modernidad exige, debido a una falla en la orientación de formación de una gran cantidad de profesionales y generación de rentabilidad económica. La cruda realidad es que tanto en las universidades, públicas y privadas del país, no 
investigan, y si lo hacen no hay una buena orientación y, por consiguiente, no producen ciencia y tecnología.

Al respecto, Ruiz-Corbella (2016) precisa: "Estamos ante la transformación de una institución de transmisora del saber, a gestora del conocimiento". Aquí está precisamente, el problema de las investigaciones de las universidades en estudio: Son exploraciones cuantitativas de una realidad, pero no presenta propuesta de cómo solucionar dichos problemas, motivo por el cual, se convierten en estudios intrascendentes.

Además, Collado (2017) está de acuerdo con el filósofo de la ciencia Paul Feyerabend (1997), quien asegura que en la educación y, por lo tanto, en la investigación no existe predominio de un paradigma o una teoría sobre otra, sino que la realidad es plural y comprende varios modelos paradigmáticos donde coexisten teorías contradictorias: conformando una realidad poliparadigmática. Teniendo en cuenta la anterior afirmación, se puede afirmar que otro de los problemas de las tesis de las universidades en cuestión, es la falta de orientación de sus estudiantes para no inclinar sus investigaciones por la metodología sólo cuantitativa (Paradigma positivista) o cualitativa (Paradigma naturalista), sino orientar los estudios por el paradigma sociocrítico.

Surge la pregunta: ¿Por qué sugerir orientar las investigaciones hacia el paradigma socio crítico? La respuesta coincide con el planteamiento de Ramos (2015), quien asegura que los aspectos que caracterizan mayoritariamente, según Escudero (1987), al paradigma crítico son: (1) poseer una visión holística y dialéctica de lo que se concibe como real, (2) la relación entre el investigador y el fenómeno de estudio se caracteriza porque todos los sujetos que participan en el proceso investigativo son activos y comprometidos con el cambio social.

Además, Escudero asegura que (3) el proceso investigativo se genera en la acción, es decir, se parte en la comprensión social de las necesidades, problemas e intereses del grupo humano que se encuentra en estudio, (4) la búsqueda de una transformación de las estructuras sociales, basada en la liberación y manumisión de los individuos que conforman el contexto social de investigación.

Cuatro características básicas que debe poseer toda investigación universitaria para cumplir la misión de la universidad de contribuir con el desarrollo de la sociedad, tal como lo establecen los diversos estatutos de las universidades del mundo, y la Ley Universitaria Peruana.

Asimismo, el docente Raúl Rivero Ayllón, docente de la universidad César Vallejo y universidad Nacional de Trujillo, indica que la razón por la cual los estudiantes se deciden por el enfoque cuantitativo es por la facilidad que encuentran a este enfoque, facilidad al momento de aplicar instrumentos que muchas veces ya se encuentran validados. Asimismo, indica que también se debe a la poca experticia de los asesores para optar por otra metodología, apoyándose en lo vertido Mamani (2019) quien afirma "que existe un vínculo entre la responsabilidad del asesor de tesis con la producción científica estudiantil".

En cuanto a las técnicas de investigación más utilizadas por los estudiantes de la UNT en la Tabla 2, tenemos a la encuesta con un $36 \%$, seguido de la observación con un $28 \%$ y la entrevista con un $20 \%$, asimismo, en la Tabla $\mathrm{N}^{\circ} 4$ podemos notar que las técnicas de investigación más utilizadas por los estudiantes de la UCV son la encuesta con un $42 \%$, seguido de la observación con un $22 \%$ y la entrevista con un $20 \%$ y en la Tabla $\mathrm{N}^{\circ} 6$ se puede verificar que las técnicas de investigación más utilizadas por los estudiantes de la UPAO son la encuesta con un $50 \%$, seguido de la observación con un $21 \%$ y la entrevista con un $20 \%$. Estos resultados concuerdan con lo también registrado por Carrión (2018) quien encuentra en su investigación que las técnicas de investigación más utilizadas por los tesistas en comunicación son la encuesta y observación, ambos con sus respectivos instrumentos como el cuestionario y guía de observación. 
Finalmente, para el procesamiento y análisis de datos el $27 \%$ de tesistas de la Universidad Nacional de Trujillo utiliza Excel y SPSS (Tabla 2), el 47\% de la Universidad César Vallejo utiliza Excel para su tratamiento, y el 46\% de la Universidad Privada Antenor Orrego utiliza Excel y el 33\% SPSS para su tratamiento. Lo anteriormente discutido explica la sobrevivencia de una metodología tradicional aún puesta en práctica por los tesistas en Ciencias de la Comunicación

En cuanto a la temática analizada en las tesis de Ciencias de la Comunicación, en la Tabla 3 , se constata que el $31 \%$ de tesis de la Universidad Nacional de Trujillo corresponden al área de comunicología y Comunicación audiovisual, el 23\% de tesis de la Universidad César Vallejo corresponde al área de diseño, publicidad y marketing y finalmente el 35\% de tesis de la Universidad Privada Antenor Orrego corresponde al área de diseño, publicidad y marketing. Estos resultados han cambiado en función a lo encontrado en años anteriores por Vera et al. (2015) quienes evidencian que las investigaciones de pregrado en la ciudad se insertan dentro de tres áreas: Marketing y publicidad, Comunicación Corporativa y Comunicación para el Desarrollo.

Por otra parte, se verifica también que la Universidad César Vallejo ha tenido un incremento en tesis de Comunicación para el Desarrollo durante los años 2017 - 2018. Esta tendencia quizá siguiendo a lo que dice Gumucio (2011), en cuanto al aporte de América Latina en los estudios de comunicación es ser pionera en la Comunicación para el Desarrollo, y como pionera en la generación tanto de prácticas concretas como de pensamiento crítico.

En esta especialidad es muy importante para el desarrollo armónico de la comunidad internacional, motivo por el cual la Organización de las Naciones Unidas (2011), ha entendido el papel que la comunicación puede desempeñar en el empoderamiento de las personas para influir sobre las decisiones que afectan a sus vidas.

Partiendo de la proposición que los estudiantes universitarios, especialmente los estudiantes que están desarrollando proyectos de investigación deben ser coherentes con la realidad en la que encuentran, y darles solución a los problemas a través de los proyectos de investigación que presentan, se hace indispensable diseñar líneas de investigación que orientan a la solución de dicha problemática, es por ello que las Universidades tanto privadas como públicas han desarrollado bajo resolución de vicerrectorado la elaboración de dichas líneas.

La Universidad Nacional de Trujillo y la Universidad Privada Antenor Orrego cuentan con resoluciones de aprobación de las líneas de investigación de la especialidad, sin embargo, ninguna de las tesis analizadas de estas casas de estudios las define en sus investigaciones, lo que podría llevar a desarrollar investigaciones mal orientadas.

Sobre las teorías en las cuales se basan los estudiantes para desarrollar sus trabajos de investigación, se observó que el $46 \%$ de los estudiantes en promedio de las 3 universidades no sustentan sus investigaciones basándose en modelos teóricos, haciéndolo solo con información referencial y conceptual, esto concuerda con las investigaciones realizadas por Lozano y Frankenber (2008) y por Vera et al. (2015) en donde encuentran que la mayoría de los estudios analizados no mencionan los fundamentos teóricos en los cuales se basan para el desarrollo de las investigaciones.

Sin embargo, se contradice con lo dicho también por Vera et al. (2015), quien indica que solo alrededor del $13 \%$ de las investigaciones utilizan como referencia o punto de partida una teoría, enfoque o modelo. De este porcentaje, el $75 \%$ corresponde a la UPAO. Las teorías predominantes son: Usos y gratificaciones, teorías sobre los efectos, Agenda setting, Difusión de innovaciones, Funcionalismo y Teoría del cultivo. Podemos notar aquí que, si bien desde hace muchos años se dejó de utilizar teorías funcionalistas, aún se siguen desarrollando en las investigaciones, teóricamente se descalifica al funcionalismo, pero en la praxis se sigue trabajando. 


\section{Conclusiones}

Una de las principales características de las tesis desarrolladas entre los años 2014 y 2018 en las tres universidades en estudio es la orientación hacia el enfoque cuantitativo, de diseño no experimental con alcance descriptivo simple, lo cual implica que la investigación sólo se centra en el aspecto teórico y no plantean soluciones concretas de los problemas que la sociedad requiere.

Otra característica similar en las tesis desarrolladas en las tres universidades es el uso las técnicas de investigación como la encuesta, y la observación siendo el cuestionario y la guía de observación los instrumentos más utilizados por los mismos, dejándose de lado otros instrumentos más confiables.

En la UNT se da mucha importancia a los temas de comunicología y comunicación audiovisual dejando de lado las otras especialidades de la carrera como son las Relaciones Públicas y la Publicidad, mientras que, en la UCV, los tesistas dan más importancia a las áreas de diseño, publicidad, marketing y comunicación audiovisual. Además la Universidad Vallejo, sí está clara la línea de investigación que desarrollan las tesis son procesos comunicaciones en la sociedad contemporánea y comunicación para el desarrollo humano, que en su mayoría no se basan en modelos teóricos, sino conceptuales; mientras que en la UPAO las áreas de diseño, publicidad y marketing y comunicación corporativa son las más utilizadas por los tesistas que no indican la línea de investigación en la cual desarrollan sus trabajos y en un gran porcentaje no se basan en modelos teóricos sino conceptuales.

En las tres Escuelas de Ciencias de la Comunicación es necesario reorientar las tesis con la finalidad de satisfacer la demanda comunicacional de la sociedad, de tal manera que los planteamientos no queden solo en propuestas, sino en desarrollo de actividades académicas, que le permitan cumplir a las universidades con su función social, y poner en práctica su Responsabilidad Social, tal como lo demanda la Ley Universitaria, teniendo en cuenta la didáctica de la investigación científica y el proceso formativo holístico e integral que necesitan los procesos formativos actuales de los profesionales en formación.

\section{Referencias}

Agudelo, N. (2004). Las líneas de investigación y la formación de investigadores: una mirada desde la administración y sus procesos formativos. Revista ieRed: Revista Electrónica de la Red de Investigación Educativa, 1(1).

Arévalo, A., \& Farné, A. (2016). Comunicación y cambio social. Un análisis desde la investigación centrado en el periodismo. Cultura, Lenguaje y representación, 15, 11-19. doi: https://dx.doi.org/10.6035/clr.2016.15.1

Bermúdez, J. (2013). Investigación científica en el Perú: factor crítico de éxito para el desarrollo del país. Sinergia E Innovación, 1(2). Recuperado de http://revistas.upc.edu.pe/index.php/sinergia/article/view/130

Calderón, G., Gutiérrez, L., \& Castaño, G. (2017). La investigación en las facultades de administración de Colombia. Revista Lasallista de Investigación, 14(1), 42-55. Recuperado en: https://www.redalyc.org/articulo.oa?id=695/69551301004

Carrión, R. (2018). Características de la estrategia metodológica de tesis de pre grado de comunicación de las universidades de Trujillo en el periodo 2011-2015 (tesis de pregrado). Universidad Nacional de Trujillo, Trujillo, Perú.

Collado, J. (2017). Reflexiones filosóficas y sociológicas de la educación: un abordaje paradigmatológico. Sophia, Colección de Filosofía de la Educación, 23, 55-82. doi: https://dx.doi.org/10.17163/soph.n23.2017.01 
Córdoba, M. (2016). Reflexión sobre la formación investigativa de los estudiantes de pregrado. Revista Virtual Universidad Católica del Norte, 47, 20-37. Recuperado de http://revistavirtual.ucn.edu.co/index.php/RevistaUCN/article/view/740/1266

Corrales, E., Reyes, J., \& Díaz, M. (2019). Asesores de tesis y publicación de artículos científicos en el pregrado. Revista Médica Herediana, 29(4), 264. doi: https://doi.org/10.20453/rmh.v29i4.3454

Denzin N.K., \& Lincoln Y.S. (Eds.) (2005). Handbook of qualitative research $\left(3^{\text {th }}\right.$ ed.) Thousand Oaks, CA.: Sage.

Elbirt, A. (2013). Comunicación para el Cambio Social y educación ambiental. Reflexiones para la construcción colectiva del desarrollo a partir de un estudio de caso en Salta, Argentina. Punto Cero, 18(26), 65-72. Recuperado de http://www.scielo.org.bo/scielo.php?script=sci_arttext\&pid=S1815$02762013000100008 \& \operatorname{lng}=\mathrm{es} \& \operatorname{tlng}=\mathrm{es}$

Escudero, J. (1987). La investigación-acción en el panorama actual de la investigación educativa: algunas tendencias. Revista de Innovación e Investigación Educativa, 3, 5-39.

Fuentes, R. (1999). Institucionalización y postdisciplinarización de las ciencias sociales en México. En: R. Fuentes \& R. Reguillo (coords.), Pensar las ciencias sociales hoy. Reflexiones desde la cultura. Guadalajara: ITESO.

García, A. (2000). Los indicadores en las políticas de la reforma universitaria argentina: balance de la situación actual y perspectiva futura. En: M. Kisilevsky (Ed.), Indicadores universitarios. Tendencias y experiencias internacionales. Buenos Aires: Eudeba.

González, G. (2013). El profesorado en formación y las finalidades de la enseñanza de las ciencias sociales. Uni-pluri/versidad. 13(2), 24-34.

González, O., \& Urbina, R. (2013). Investigación universitaria herramienta de transformación social. Formación Gerencial 12(2), 193-210. Recuperado de https://dialnet.unirioja.es/servlet/articulo?codigo $=4772669$

Grinnell, R. (1997). Social work research \& evaluation: Quantitative and qualitative approaches $\left(5^{\text {th }}\right.$ ed.). Illinois: E.E. Peacock Publishers.

Gumucio, A. (2011). Comunicación para el cambio social: clave del desarrollo participativo. Signo y Pensamiento, $X X X$ (58), 26-39. Recuperado de en: https://www.redalyc.org/articulo.oa?id=860/86020038002

Gutiérrez, I., Benítez, H., \& González, H. (2018). Integración de la investigación y la enseñanza en las universidades médicas. Educación Médica. doi: https://dx.doi.org/10.1016/j.edumed.2018.07.007.

Hernández, R., Fernández, C., \& Baptista, P. (2014). Metodología de la Investigación (6 ${ }^{\mathrm{a}}$ ed.). México D.F.: McGraw-Hill Interamericana.

Huaraca, C., Apaza, A. \& Mejía, C. (2017). Realidad peruana de la publicación científica estudiantil en los últimos diez años. Educación Médica Superior, 31(3), 124-134. Recuperado de http://scielo.sld.cu/scielo.php?script=sci_arttext\&pid=S086421412017000300014\&lng=es\&tlng=es.

Ibáñez, M., Morresi, S., \& Delbianco, F. (2017). Una medición de la eficiencia interna en una universidad argentina usando el método de fronteras estocásticas. Revista de la educación superior, 46(183), 47-62. Recuperado de http://resu.anuies.mx/ojs/index.php/resu/article/view/25

Igartua, J.J., \& Humanes M.L. (2004). El método científico aplicado a la investigación en comunicación social. Portal de la comunicación INCOM UAB.

Larrán, M., \& Andrades, F. (2015). Análisis de la responsabilidad social universitaria desde diferentes enfoques teóricos. Revista Iberoamericana de Educación Superior, 6(15), 91- 
107.

Recuperado

de

http://www.scielo.org.mx/scielo.php?script=sci_arttext\&pid=S2007-

$28722015000100005 \& \operatorname{lng}=$ es\&tlng=es.

Ley N. ${ }^{\circ} 30220$, Ley Universitaria (2014). En Diario Oficial El Peruano.

Lozano, J. \& Frankenberg, L. (2008). Enfoques teóricos y estrategias metodológicas en la investigación empírica de audiencias televisivas en América Latina: 19922007. Comunicación y Sociedad, 10, 81-110. Recuperado de https://www.redalyc.org/articulo.oa?id=346/34601004

Mamani, J. (2019). La asesoría de tesis en pregrado: una labor que requiere un nuevo enfoque. Revista Médica Herediana, 30(2), https://dx.doi.org/https://doi.org/10.20453/rmh.v30i2.3555

Memorias del Congreso Iberoamericano de Ciencia, Tecnología, Innovación y Educación. Buenos Aires, Argentina, 12, 13 y 14 de noviembre de 2014. Recuperado de https://www.oei.es/historico/congreso2014/memorias2014.php

Miyahira, J. (2009). La investigación formativa y la formación para la investigación en el pregrado. Revista Médica Herediana, 20(3), 119-122. doi: https://dx.doi.org/10.20453/rmh.v20i3.1010

Navarro, R. (2011). 50 años de investigaciones de la Comunicación en México: un recuento descriptivo de la producción publicada. Intercom: Revista Brasileira de Ciências da Comunicação, 34(1), 213-231. doi: https://dx.doi.org/10.1590/S1809-58442011000100011

ONU (2011). Comunicación para el Desarrollo. Fortaleciendo la eficacia de las Naciones Unidas. Programa de las Naciones Unidas para el Desarrollo. Nueva York.

Pereira, Z. (2011). Los diseños de método mixto en la investigación en educación: Una experiencia concreta. Revista Electrónica Educare, XV(1),15-29. Recuperado de https://www.redalyc.org/articulo.oa?id=1941/194118804003

Pétriz, F., \& Rubiralta, M. (2017). Las misiones de la universidad. Cuadernos de Pedagogía, 476, 46- 50.

Pineda, M. (2006). La investigación de la comunicación en América Latina: Evaluación del estado de la cuestión. Opción, 22(50), 142-158. Recuperado de http://ve.scielo.org/scielo.php?script=sci_arttext\&pid=S1012$15872006000200008 \& \operatorname{lng}=$ es\&tlng=es.

Ramírez, C., García, E. \& Cruel, J. (2017). Gestión educativa y desarrollo social. Dominio de las Ciencias, 3(1), 378-390. Recuperado de http://dominiodelasciencias.com/ojs/index.php/es/article/view/801

Ramos, C. (2015). Los paradigmas de la investigación científica. Avances en psicología, 23(1), 12- 31.

Roque, Y., Alonso, S., \& Maldonado, A. (2019). Nivel de satisfacción con la estrategia de investigación científica en una facultad de la Universidad Nacional de Chimborazo, Ecuador. IE Revista de Investigación Educativa de la REDIECH, 10(18), $\quad 177 \quad$ - $191 . \quad$ Recuperado de https://www.rediech.org/ojs/2017/index.php/ie_rie_rediech/article/view/522

Ruiz, M., \& López, E. (2019). La misión de la universidad en el siglo XXI: comprender su origen para proyectar su futuro. Revista de la educación superior, 48(189), 119.

Ruiz, M. (2016). La universidad ante los nuevos escenarios virtuales de aprendizaje. En: M. A. Santos Rego (Ed.), Sociedad del Conocimiento. Aprendizaje e Innovación en la Universidad (pp. 91-114). Madrid: Biblioteca Nueva.

Sánchez, J., Sánchez, I., \& Viloria, J. (2016). Problemas y necesidades en la gestión de las organizaciones educativas: implicaciones para la formación avanzada de los 
docentes y directivos docentes del departamento del Magdalena. En: R. Muñoz, Administración y estudios organizacionales. Complementariedades $y$ contradicciones. Medellín: Red Pilares.

Sánchez, J., Sánchez, I., \& Viloria, J. (2017). Expectativas de formación avanzada en el campo de la gestión educativa: una lectura desde las voces de directivos docentes en el departamento del Magdalena, Colombia. En: Herrera, D., Ramírez, G., y Rosas, J. (Ed), Diversidad y Complejidad Organizacional en América Latina (pp. 21-60). Recuperado de http://remineo.org/repositorio/libros/dcoal/wp content/uploads/2017/08/12-Territorio-yorganizaciones.pdf\#page $=21$

Tena, D., (2017). Diseño gráfico publicitario. Diseño gráfico y dirección de arte publicitaria. Madrid: Síntesis.

Tobón, S. (2013). Socioformación: hacia la gestión del talento humano acorde con la sociedad del conocimiento. México: CIFE.

Unesco (1998). Declaración Mundial sobre la Educación Superior en el Siglo XXI: visión y acción. Marco de acción prioritaria para el cambio y el desarrollo de la educación superior. París: UNESCO. Recuperado de http://www.unesco.org/education/educprog/wche/declaration_spa.htm.

Vera, R., Castañeda, K., \& Chirinos, S. (2015). Historia de la investigación en comunicación social, nivel de pregrado en Trujillo. Pueblo continente, 26(1). Recuperado de http://journal.upao.edu.pe/PuebloContinente/article/view/299/267

Vergara, M. (2011). Funciones ejecutivas y desempeño académico en estudiantes de primer año de psicología de la corporación universitaria minuto de dios, en Bello Antioquia (tesis de maestría). Universidad San Buenaventura, Bogotá, Colombia. Recuperado de http://hdl.handle.net/10819/1219 